\title{
O ESTUDO DA VIABILIDADE TÉCNICO-ECONÔMICA PARA RESFRIAMENTO UTILIZANDO EJETORES A VAPOR
}

\author{
L. Y. A. SILVA ${ }^{1}$, D. Y. FUCASSE ${ }^{1}$, K. S. SANTOS ${ }^{1}$ e R. NASSER JUNIOR ${ }^{1}$ \\ ${ }^{1}$ Universidade Federal de São Paulo, Instituto de Ciência Ambientais, Químicas e Farmacêuticas, \\ Departamento de Ciências Exatas e da Terra \\ E-mail para contato: roberto.nasser@unifesp.br
}

\begin{abstract}
RESUMO - Nas indústrias, os sistemas de resfriamento e aquecimento consomem grande quantidade de energia, gerando altos custos, devido ao preço elevado da energia elétrica, sendo necessárias melhorias na área de eficiência energética. A maioria das indústrias utiliza o ciclo de refrigeração por compressão a vapor de fluidos térmicos, o que gera um alto consumo de energia elétrica. Uma opção aos ciclos de refrigeração por compressão a vapor é o uso dos ejetores a vapor. O ejetor é um equipamento sem partes móveis, de operação simples, que pode ser usado para gerar vácuo, e que utiliza vapor como fluido motriz. Assim, o vapor que é descartado em uma etapa da unidade pode ser reutilizado em sistemas de refrigeração com ejetores, o que diminui o consumo de energia elétrica e reduz a emissão de vapor ao ambiente. A viabilidade técnico-econômica da substituição do sistema de refrigeração por compressão a vapor no processo de resfriamento da água por um sistema com ejetor a vapor foi avaliado. O estudo usou dados de uma indústria têxtil e informações do fornecedor do ejetor e foi constatado que existe a viabilidade econômica para a substituição com uma amortização do investimento inferior a 3 anos.
\end{abstract}

\section{INTRODUÇÃO}

Nas indústrias químicas uma variável de processo muito importante, além da matéria-prima e da mão de obra, é a energia. Considerando o atual crescimento da indústria brasileira e do custo elevado da energia elétrica na produção industrial, faz-se cada vez mais necessário o estudo da eficiência energética para a redução dos custos e otimização de processos.

Nas diferentes indústrias, os sistemas de resfriamento e aquecimento normalmente trabalham na sua potência máxima, consumindo grande quantidade de energia. A maioria das indústrias no Brasil utiliza o ciclo de refrigeração por compressão a vapor, que apesar da eficiência do processo, apresenta elevado consumo de energia elétrica.

Uma alternativa aos ciclos de refrigeração por compressão a vapor é o uso dos ejetores a vapor. As principais vantagens do ejetor são: a) é um equipamento simples, sem partes móveis e com baixo custo de manutenção; b) água na forma de vapor pode ser utilizada como fluido de 
trabalho (He et al., 2009).

Os principais componentes de um ejetor são: o bocal principal, a câmara de sucção, a câmara de mistura e o difusor, como pode ser visto na Figura 1.

$\mathrm{Na}$ entrada, a corrente do fluido primário é expandida e acelerada através de um bocal convergente-divergente, atingindo a velocidade supersônica; formando uma região de baixa pressão na saída do bocal que arrasta o fluido secundário. Na câmara de mistura os fluidos primário e secundário são completamente misturados, e no fim da câmara de mistura a velocidade de escoamento é supersônica. Uma onda de choque é então produzida dentro da seção de área constante, criando um efeito de compressão, e a velocidade de escoamento é reduzida a um valor subsônico. Após a compressão do fluido ser atingida, as correntes combinadas dos fluidos primário e secundário escoam através do difusor (Chen et al., 2013).

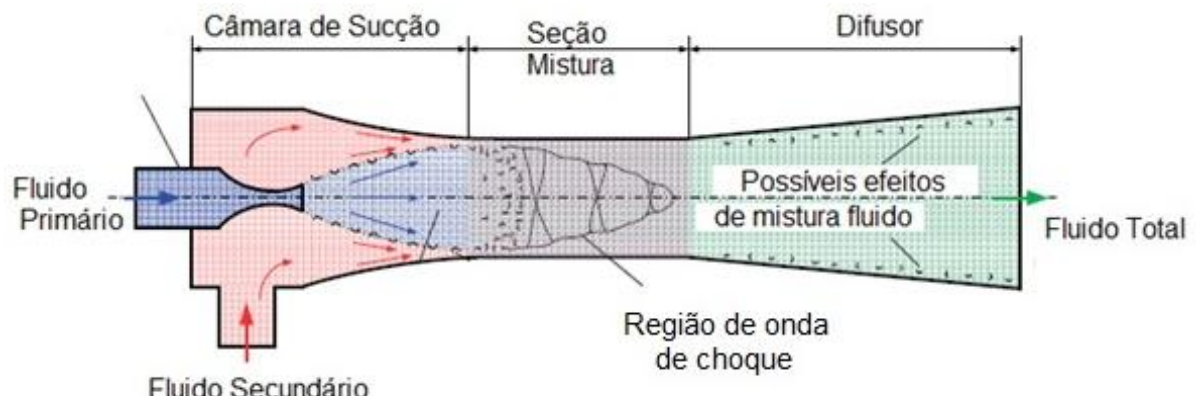

Figura 1 - Esquema do Ejetor (Elbel e Hrnjak, 2008).

O ciclo termodinâmico do sistema de refrigeração com ejetor a vapor está representado na Figura 2. Calor é adicionado ao gerador, onde vapor a alta pressão e temperatura é produzido e usado como fluido primário no ejetor. $\mathrm{O}$ ejetor arrasta o fluido a baixa pressão (fluido secundário) do evaporador. Isto faz com que o fluido secundário evapore a uma baixa pressão e produz o efeito de refrigeração. $\mathrm{O}$ vapor que sai do ejetor é encaminhado para o condensador onde ele é liquefeito a temperatura ambiente. Parte do líquido é bombeada novamente para o gerador, enquanto o restante passa através de uma válvula de expansão e retorna ao evaporador (Chunnanond e Aphornratana, 2004). 


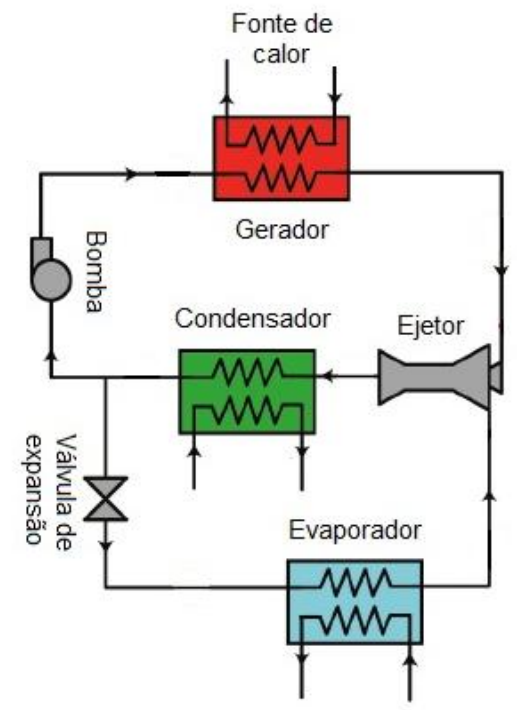

Figura 2 - Ciclo de Refrigeração com Ejetor a Vapor (Little e Garimella, 2011).

Com os atuais problemas ambientais, e a busca das indústrias por otimização de processos, visando qualidade, eficiência e baixo custo, justifica-se a avaliação técnico-econômica de ejetores.

Este trabalho tem por objetivo executar o estudo da viabilidade técnica e econômica da aplicação de ejetores a vapor em um processo de resfriamento de água de uma indústria têxtil. Em uma das etapas do processo produtivo desta indústria existe o descarte de vapor de 10,81 bar, que pode ser reutilizado como fluido primário no sistema de refrigeração com ejetor a vapor para resfriar uma corrente de água de $12{ }^{\circ} \mathrm{C}$ para $7{ }^{\circ} \mathrm{C}$.

\section{MATERIAIS E MÉTODOS}

\subsection{Balanço de Massa e Energia}

Para determinar a quantidade de vapor de sucção (fluido secundário) para refrigerar uma corrente de $88,00 \mathrm{~kg} / \mathrm{h}$ de água de $12{ }^{\circ} \mathrm{C}$ para $7{ }^{\circ} \mathrm{C}$ realizou-se os balanços de massa e energia no evaporador que estão representados pelas Equações 1 e 2. De acordo com a Figura 3, no evaporador estão presentes três correntes: $m_{1}$ (corrente de água a $12{ }^{\circ} \mathrm{C}$ ), $m_{2}$ (corrente de vapor d'água - fluido secundário) e $m_{3}$ (corrente de água a $7{ }^{\circ} \mathrm{C}$ ).

$$
m_{1}=m_{2}+m_{3}
$$




$$
\dot{m}_{1} \cdot \hat{H}_{1}=\dot{m}_{2} \cdot \hat{H}_{2}+\dot{m}_{3} \cdot \hat{H}_{3}
$$

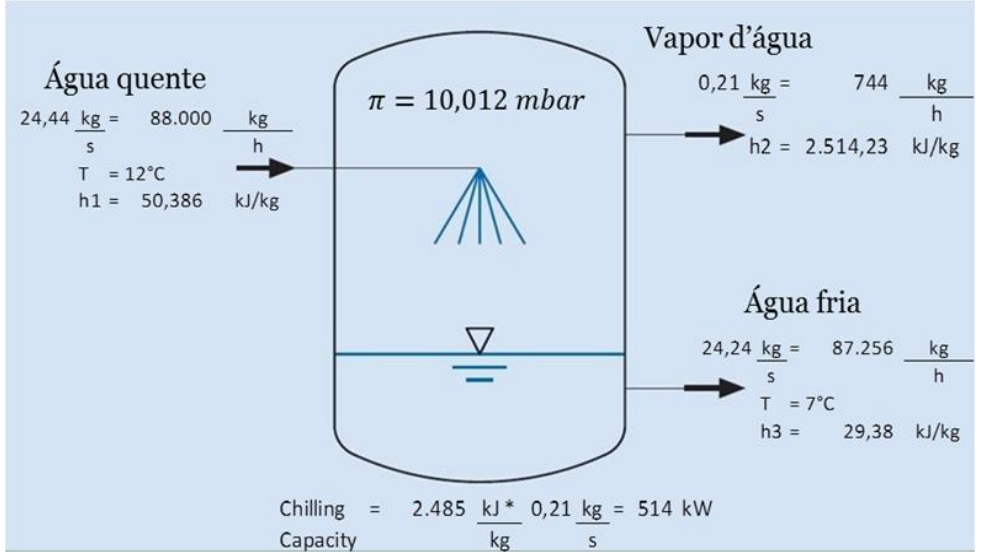

Figura 3 - Esquema do Evaporador.

A Tabela 1 apresenta os valores da vazão mássica $(\dot{m})$ e da entalpia $(H)$ de cada corrente do evaporador.

Tabela 1 - Vazões mássicas e entalpias do evaporador

\begin{tabular}{|c|c|c|}
\hline Corrente & $\dot{m}(\mathrm{~kg} / \mathrm{h})$ & $\hat{H}(\mathrm{~kJ} / \mathrm{kg})$ \\
\hline 1 & 88000 & 50,39 \\
\hline 2 & 744,00 & 2514 \\
\hline 3 & 87256 & 29,38 \\
\hline
\end{tabular}

\subsection{Determinação da Quantidade de Vapor Motriz (Fluido Primário)}

Existem vários parâmetros usados para descrever o desempenho de um ejetor. Para aplicações em refrigeração, os parâmetros mais importantes são: a razão de arraste $(\mathrm{Rm})$, a razão de compressão (K) e a razão de expansão (E). A razão de arraste é a razão entre a vazão do fluido secundário e a vazão do fluido primário, este parâmetro está relacionado com a eficiência do ciclo de refrigeração. A razão de compressão é a razão entre a pressão de descarga e a pressão de sucção do ejetor enquanto que a razão de expansão é a razão entre a pressão do fluido primário e a pressão de sucção, estes parâmetros limitam a temperatura no qual o calor do ciclo de refrigeração pode ser rejeitado (Chunnanond e Aphornrata, 2004 e Chen et al., 2013). 
O vapor motriz (fluido primário) que foi utilizado neste estudo foi o vapor de 10,81 bar disponível na indústria cujos dados são a base deste estudo. Assim, o valor da razão de expansão (E) para o caso estudado é igual a:

$$
E=\frac{\text { pressão do fluido primário }}{\text { pressão de sucção }}=\frac{10810 \text { mbar }}{10 \text { mbar }}=1081
$$

Para determinar a razão de compressão $(\mathrm{K})$ é necessária a pressão de descarga do ejetor. A pressão de descarga depende da razão de arraste $(\mathrm{Rm})$. A dependência é tal que a razão de arraste e a pressão de descarga são inversamente dependentes. Quanto menor a razão de arraste, maior a pressão de descarga e menor é o coeficiente desempenho do sistema de refrigeração (Sokolov e Hershgal, 1990). Com o objetivo de aumentar o valor de Rm, diminuindo a quantidade de vapor motriz, escolheu-se a pressão de descarga de 30 mbar, que resulta em uma razão de compressão de:

$$
K=\frac{\text { pressão de descarga }}{\text { pressão de sucção }}=\frac{30 \text { mbar }}{10 \text { mbar }}=3
$$

Com auxílio da Figura 4, determinou-se o coeficiente b, que é o inverso de Rm, igual a 0,8. Assim, a quantidade de vapor motriz é dada por:

$$
b=\frac{1}{R m}=\frac{\text { vazão do fluido primário }}{\text { vazão do fluido secundário }}=\frac{\text { vazão do fluido primário }}{744,00}=0,8
$$

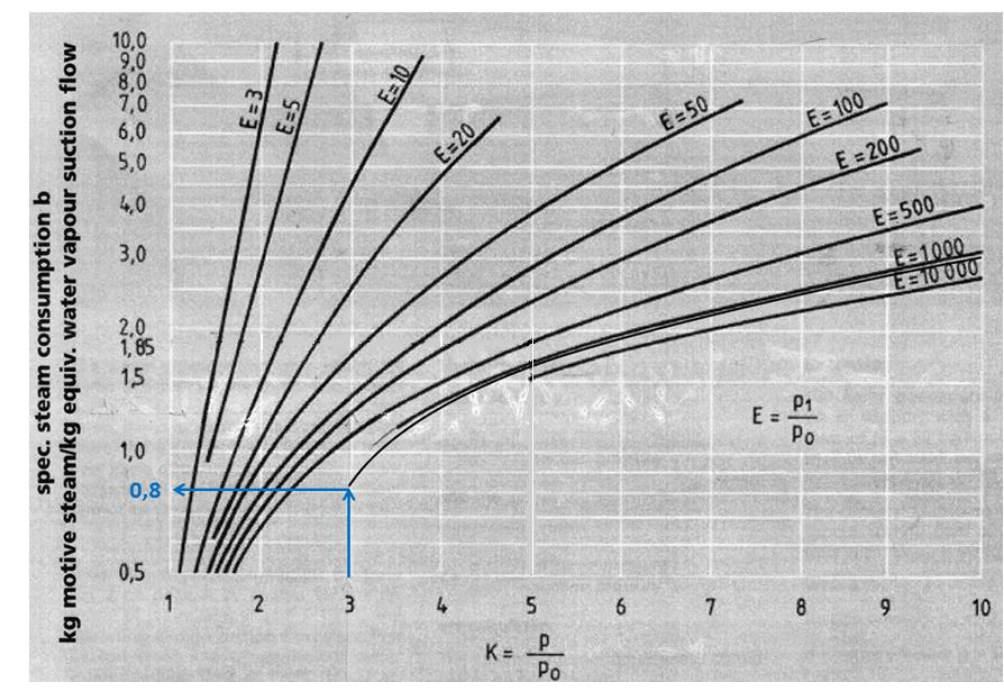

Figura 4 - Coeficiente específico de vapor consumido (GEA, 2009).

Obtém-se uma vazão de vapor motriz igual a $595 \mathrm{~kg} / \mathrm{h}$. 


\subsection{Análise da Viabilidade Econômica}

De acordo com Perlingeiro (2008), o Investimento Total ( $\left.\mathrm{I}_{\text {total }}\right)$ de um projeto é a soma de todos os recursos alocados em um empreendimento. $\mathrm{I}_{\text {total }}$ é dado pelo Investimento Fixo ( $\mathrm{I}_{\text {fixo }}$ ), o Capital de Giro ( $\mathrm{I}_{\text {giro }}$ ) e o Investimento inicial $\left(\mathrm{I}_{\text {partida }}\right)$. O investimento fixo compreende $\mathrm{O}$ Investimento Direto $\left(\mathrm{I}_{\text {direto }}\right)$, material para montagem da instalação, e o Investimento Indireto ( $\mathrm{I}_{\text {indireto }}$ ), assim como o investimento ISBL (dentro dos limites de bateria), e o investimento OSBL (fora dos limites de bateria), ou seja, investimentos para aquisição, transporte, instalação, serviços de engenharia, entre outros.

$$
I S B L=f_{T} \cdot f_{D} \cdot f_{L} \cdot \sum I_{e i}
$$

Em que $I_{e i}$ é o preço de compra do equipamento em data e local definidos; $f_{T}$ é o fator empírico de transferência do equipamento (onde foi fabricado, para o local de construção da planta - inclui custos de transporte, alfândega, armazenamento, seguros); $f_{D}$ é o fator de atualização de preço para o ano vigente e $f_{L}$ é o fator de Lang, que relaciona o percentual de acréscimo referentes a natureza física e despesas adicionais a instalação dos equipamentos.

A estimativa de investimento $\left(\mathrm{I}_{\mathrm{ei}}\right)$ é dada pela utilização de um fator, no qual relaciona custos e capacidades:

$$
I_{e i}=I_{e b i}\left(\frac{Q_{i}}{Q_{b i}}\right)^{M_{i}}
$$

No qual $I_{e i}$ é o valor estimado do equipamento i para a dimensão ou capacidade $Q_{i} ; I_{e b i}$ representa o valor estimado do equipamento i para a dimensão ou capacidade $Q_{b i}$ e $M_{i}$ é o fator experimental de escala para o equipamento $i$.

O tempo de recuperação do investimento, também conhecido como "payback", é dado por,

$$
T R C=\frac{I-(A-I)}{C P_{A}-C P_{i}}
$$

Em que $I$ é o investimento do equipamento alternativo; $A$ é o investimento de um equipamento similar ao atual; $C P_{A}$ é o custo do processo alternativo e $C P_{i}$ é o custo do processo atual.

\section{RESULTADOS E DISCUSSÕES}

O consumo de vapor motriz calculado em 2.2 é muito inferior ao disponível como sobra na instalação, o que comprovou a viabilidade técnica da proposta. 


\section{9 a 22 de outubro de 2014 \\ Florianópolis/SC}

Os dados históricos de consumo de energia elétrica fornecidos mostraram que, para 2013 o valor foi de $613.442 \mathrm{kWh} / \mathrm{mês}(2.208 .391 \mathrm{MJ} / \mathrm{mês})$, correspondendo a um custo mensal de $\mathrm{R} \$$ 127.377. O vapor consumido na planta representa uma energia de $7.001 .434 \mathrm{MJ} / \mathrm{mês,}$ correspondendo a um custo mensal de $\mathrm{R} \$ 214.983$. Portanto, o custo da energia elétrica é 0,058 $\mathrm{R} \$ / \mathrm{MJ}$ e do vapor $0,031 \mathrm{R} \$ / \mathrm{MJ}$, ou seja, custo do vapor motriz é $47 \%$ menor que o custo da energia elétrica.

O gasto energético para a vazão calculada de vapor motriz nos ejetores $595 \mathrm{~kg} / \mathrm{h}$, considerando utilização continua, totalizando 8.000 h/ano, e uma entalpia da água a 11 bar de $1.999 \mathrm{~kJ} / \mathrm{kg}$, resulta em um gasto energético de 9.518.292 MJ/ano, representando uma economia energética de 876.724 MJ/ano, se comparada com a atual utilização dos compressores. Haveria um gasto energético menor combinado a um custo também menor, devido ao preço do vapor mais barato, obtendo uma economia $\mathrm{kR} \$ 307$ por ano.

Após a comprovação econômica da utilização do ejetor, foi efetuada uma estimativa de custo por meio de índices, com base em um fornecimento anterior, de valor $\mathrm{kR} \$ 4.223$, para uma capacidade térmica distinta.

Utilizando a Equação 6, considerando uma taxa de juros conservadora de 1,75\% a.m., cobrado pelas instituições bancárias para financiar projetos e um período de 48 meses, o valor orçado foi para o valor presente, obtendo-se R 9.711 (Pelingeiro, 2008).

A equalização de custos devido às distintas capacidades térmicas foi realizada pela utilização da Equação 7, estimando o custo real do ejetor pela razão das capacidades térmicas elevada ao coeficiente de Lang, neste caso 0,67 (Pelingeiro; 2008), obtendo kR\$2.723.

Para obter o TRC do projeto, leva-se em consideração a troca do atual compressor existente na planta. Baseando-se em um orçamento anterior de um compressor de similar robustez, o valor presente de seu custo, considerando uma taxa de juros nominal de 1,75\% a.m. por um período de 171 meses, é kR\$10.809.

Foram então propostos dois cenários: o primeiro, no qual seria substituído por um compressor de igual robustez ao atual, e segundo, no qual o compressor teria capacidade térmica similar ao do ejetor. Para o primeiro cenário, calculando-se o TRC pela equação 8 , considerando a simples economia pontual dada pela diferença entre o custo dos ejetores e o compressor, torna a amortização da proposta imediata. $\mathrm{O}$ segundo cenário exige a adequação do orçamento do compressor (de $6.750 \mathrm{MJ} / \mathrm{h}$ ) para a capacidade térmica do ejetor, de $1.841 \mathrm{MJ} / \mathrm{h}$. Elevando-se a razão da capacidade calorífica ao mesmo coeficiente de Lang $(0,67)$, e multiplicando-se o valor do orçamento para o compressor já trazido a valor presente resulta em $\mathrm{kR} \$ 4.526$.

Por fim, neste segundo cenário, o $\mathrm{TRC}=2,99$ anos, considerando-se uma economia pontual de kR\$1.803, e uma economia de custo ao longo do ano, decorrente ao menor gasto energético de $\mathrm{kR} \$ 307$. 
Vale ressaltar ainda que esse valor é pontual para este caso, e em condições mais favoráveis, no qual o custo da energia elétrica seja mais cara, a exemplo de regiões remotas que utilizam geradores a diesel, e haja um excedente de vapor de escape, como observado nas indústrias sucroalcooleiras, o payback poderá ser mais atrativo.

\section{CONCLUSÕES}

Conclui-se que a proposta é técnica e economicamente viável, apresentando um TRC máximo de 3 anos, no caso mais conservador.

\section{REFERÊNCIAS}

CHEN, X.; OMER, S.; WORALL, M.; RIFFAT, S. Recent developments in ejector refrigeration Technologies. Renew. Sust. Energ. Rev., v. 19, p. 629-651, 2013.

CHUNNANOND, K.; APHORNRATANA, S. Ejectors: applications in refrigeration technology. Renew. Sust. Energ. Rev., v. 8, p. 129-155, 2004.

HE, S,; LI, Y.; WANG, R. Z. Progress of mathematical modeling on ejectors. Renew. Sust. Energ. Rev., v.13, p. 1760-1780, 2009.

ELBEL, S.; HRNJAK, P. Ejector refrigeration: an overview of historical and present developments with an emphasis on air-conditioning applications. International Refrigeration and Air Conditioning Conference, paper 884, 2008.

LITTLE, A. B.; GARIMELLA, S. A Review of ejector technology for refrigeration applications. IJACR, v. 19, p.1-15, 2011.

GEA Cooling under vacuum. Ettlingen, Alemanha, 2009.

PERLINGEIRO, C. A. Engenharia de Processos - Análise, Simulação, Otimização e Síntese de Processos Químicos, $1^{\mathrm{a}}$ ed., Rio de Janeiro, Editora Bluchner, 2008.

SOKOLOV, M.; HERSHGAL, D. Enhanced refrigeration cycles powered by low grade heat. Part 1. Systems characterization. Int. J. Refrig, v. 13, p.351-356, 1990.

WOLK, Wolfgang. Steam jet refrigeration plants and their range of applications. British Chemical Engineering, v. 3, 1969. 